\title{
Optically controlled delays for broadband pulses
}

\author{
Qingqing Sun, ${ }^{1}$ Yuri V. Rostovtsev, ${ }^{1}$ Jonathan P. Dowling, ${ }^{1,2}$ Marlan O. Scully, ${ }^{1,3}$ and M. Suhail Zubairy ${ }^{1}$ \\ ${ }^{1}$ Department of Physics and Institute of Quantum Studies, Texas A\&M University, College Station, Texas 77843-4242, USA \\ ${ }^{2}$ Hearne Institute for Theoretical Physics, Department of Physics and Astronomy, Louisiana State University, Baton Rouge, Louisiana \\ 70803-4001, USA \\ ${ }^{3}$ Princeton Institute for Materials Research, Princeton University, Princeton, New Jersey 08544-1009, USA
}

(Received 3 May 2005; published 19 September 2005)

\begin{abstract}
We propose a scheme that provides large controllable delays for broadband optical pulses. The system is based on the steep dispersion of a coherently driven medium, in which the narrow electromagnetically induced transparency (EIT) band is overcome by using spatial and temporal processing.

DOI: 10.1103/PhysRevA.72.031802

PACS number(s): 42.50.Gy, 42.65.Re
\end{abstract}

Recent progress in the study of ultra-short optical pulse generation [1] creates a fundamentally new realm of laser applications in many areas, including material science, information processing, communication and spectroscopy. The rapidly developing technology of broadband optical pulse shaping requires systems to provide controllable delays for such pulses. For example, an optical buffer [2] can be characterized by the maximum number of bits $N_{b}$ that can be simultaneously stored in the buffer.

In this paper, we show that the steep dispersion of an electromagnetically induced transparency (EIT) medium $[3,4]$ can be used to create large controllable delays for ultrashort pulses by using the system shown in Fig. 1. In particular we show that it is possible to produce a 10 microseconds delay for $10 \mathrm{ps}$ optical pulses, thus yielding a timedelay-bandwidth product of about $10^{6}$. The best product achieved so far in slow-light experiments is 3 [5-7]. An important feature of our scheme is that the delay is continuously controllable by an optical field. The idea is to synthesize dispersion of the system by using the highly steep dispersion of a three-level atomic system with inhomogeneous broadening.

In Fig. 1 we present two possible schemes. The system consists of a set of prisms (or diffraction gratings) with total dispersion equal to zero. The first prism disperses the probe pulse transforming the parallel beam into divergent beams. The angle of refraction of the light beam with frequency $\omega_{L}$ is $\beta\left(\omega_{L}\right)=\left[n\left(\omega_{L}\right)-1\right] \alpha[8]$, where $n\left(\omega_{L}\right)$ is the refractive index of the material of the prism [see Fig. 2(A)]. The second prism produces parallel beams with different frequencies shifted in space [see Figs. 2(A) and 2(B)]. The light beams of varying frequencies then pass through an atomic medium that has an inhomogeneous magnetic field gradient [see Fig. 2(C)]. The medium consists of a three-level atomic system. A drive field couples the levels $a$ and $c$ and the probe field couples levels $a$ and $b$.

In the first scheme, we maintain one- and two-photon resonances of the driving and probe fields at the $a-c$ and $a$ $-b$ transitions, respectively, by an appropriate design of the prism system. This scheme can work for hot gases with Doppler broadened transitions. The second scheme has a driving field that propagates in the perpendicular direction to the probe field. Here the medium should not have Doppler broadening, i.e., this scheme works for cold atoms. Also it assumes that the $a-c$ transition is not influenced by the magnetic field at all locations inside the medium, and the driving field is always at resonance [see Fig. 2(C)]. A similar scheme has been presented in [9] and a scheme based on photorefractive materials has also been discussed in [10].

We assume a weak probe and a strong resonant drive field, i.e., all population is in state $b$. The susceptibility $\chi$ associated with the $a-b$ transition has the form [4]

$$
\chi\left(\nu_{p}\right)=\frac{-\eta \gamma\left(\Delta_{p}-i \gamma_{c b}\right)}{\left(\gamma_{c b}+i \Delta_{p}\right)\left(\gamma_{a b}+i \Delta_{p}\right)+|\Omega|^{2}},
$$

where $\Delta_{p}=\omega_{a b}-\nu_{p}$ is the detuning of the probe from the atomic transition $a-b ; \nu_{p}$ is the frequency of the probe field $E_{p} ; \Omega$ is the Rabi frequency of the drive field on the $a-c$ transition, $\gamma_{a b}$ and $\gamma_{c b}$ are the decay rates of the atomic coherences $\rho_{a b}$ and $\rho_{c b}$, respectively; $\gamma$ is the radiation decay

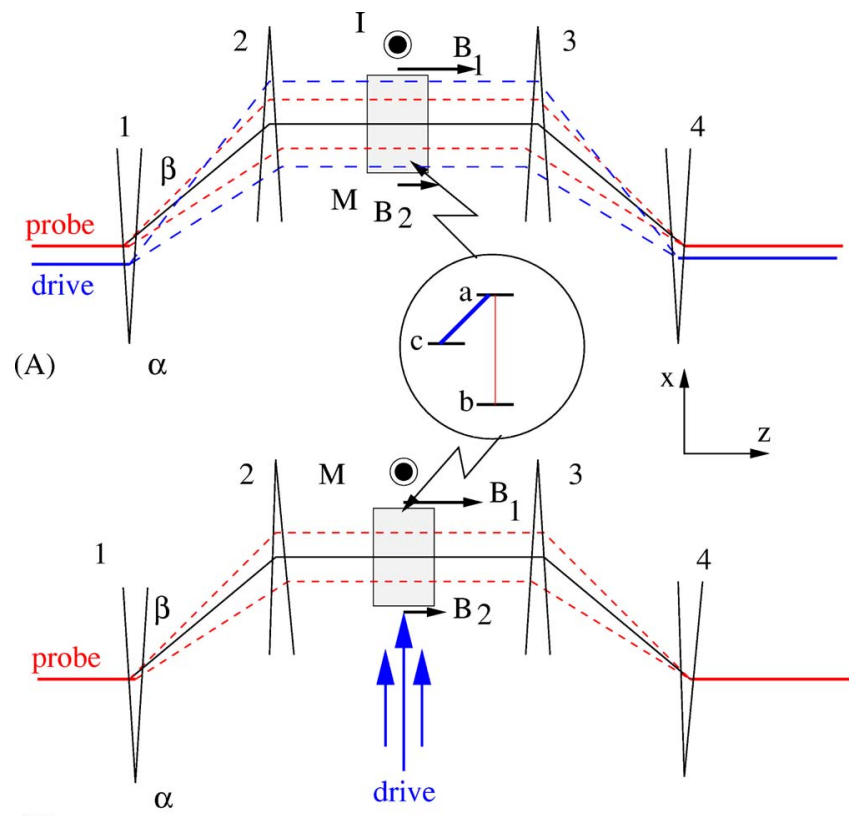

(B)

FIG. 1. (Color online) Schemes providing a continuous controllable delay to a broadband optical pulse. 

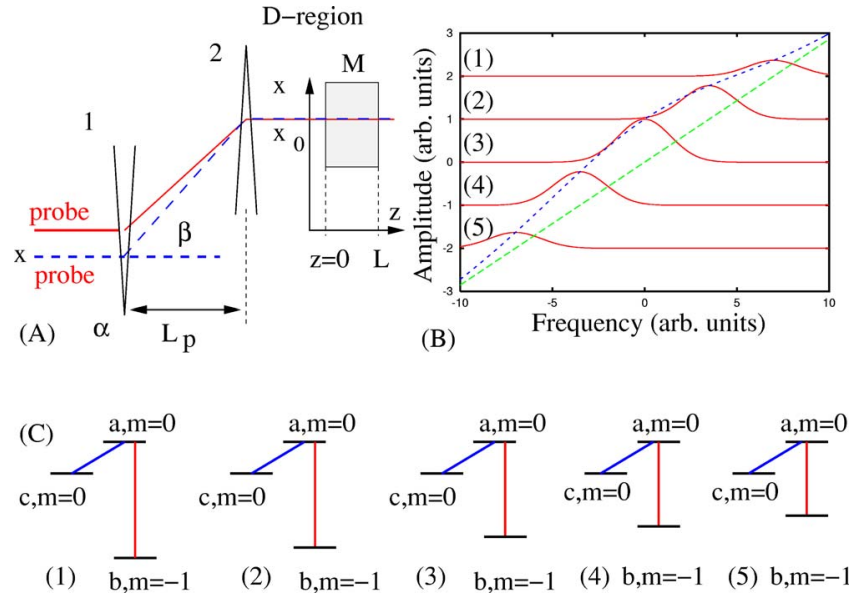

$\begin{array}{lll}\text { (3) } b, m=-1 & \text { (4) } b, m=-1 & \text { (5) } b, m=-1\end{array}$

FIG. 2. (Color online) (A) The beam of light has a Gaussian profile along $x$ axis. Each ray in the dispersion region consists of all frequencies $\omega_{x}$ that come from the transverse rays dispersed by the prisms. The solid line corresponds to the center of the probe beam, the dashed line is shifted at the distance $x$ from the center of the beam. The D-region is the dispersive region where different frequencies of the probe pulse are separated. (B) The spectra of the probe pulse are shown in different locations of the D-region (1-5). The dashed lines show the positions of the maximum and the maximum amplitudes of the probe pulses correspondingly, visualizing the spectral distribution of the probe pulse along the $x$-axis. The total width of the spectra is determined by the duration of the probe pulse. The spectral width of the probe pulse at a particular location is determined by the frequency dispersion of the system of prisms. (C) A three-level atomic medium with inhomogeneous magnetic field. It is shown that at different locations inside medium (1-5) the probe transition has different frequency, $\omega_{a b}=\omega_{a b 0}+\alpha x$, that are in resonance with the corresponding frequency component of the probe field, $\omega_{L}=\omega_{0}+\beta x$, via the system of prisms shown in Fig. 1 .

between $a$ and $b$, and $\eta=3 \lambda^{3} N / 8 \pi^{2}$ with $\lambda$ being the wavelength of the probe field and $N$ being the atomic density. In hot gases, the Doppler shift changes the detuning $\Delta_{p} \rightarrow \Delta_{p}$ $+k v$ ( $k$ is the wave vector) due to thermal motion. For the sake of simplicity we consider a Lorentzian profile with Doppler width $D$ for the inhomogeneously broadened line shape $F(v)$. Averaging over inhomogenous distribution, i.e., $\langle\chi\rangle_{v}=\int_{-\infty}^{\infty} d v \chi F(v)$, gives

$$
\chi\left(\nu_{p}\right)=\frac{-\eta \gamma\left(\Delta_{p}-i \gamma_{c b}\right)}{\left(\gamma_{c b}+i \Delta_{p}\right)\left(\gamma_{a b}+D+i \Delta_{p}\right)+|\Omega|^{2}} .
$$

The real part of $\chi$ is given by

$$
\chi^{\prime}=\frac{-\eta \gamma \Delta_{p}\left(\Omega^{2}-\gamma_{c b}^{2}-\Delta_{p}^{2}\right)}{\left(\Omega^{2}+\gamma_{c b} D-\Delta_{p}^{2}\right)^{2}+\Delta_{p}^{2}\left(\gamma_{c b}+D\right)^{2}} .
$$

In general, a pulse propagating in the resonant medium is distorted because $\chi^{\prime}$ has nonvanishing higher-order derivatives with repect to $\nu_{p}$, and the group velocity for the different frequency components of a pulse are different. We first address the question: What is the largest delay and the shortest pulse that can propagate through this EIT medium without distortion? This question was discussed in Refs. [11,12]. We shall then analyze the advantages of inhomogeneous
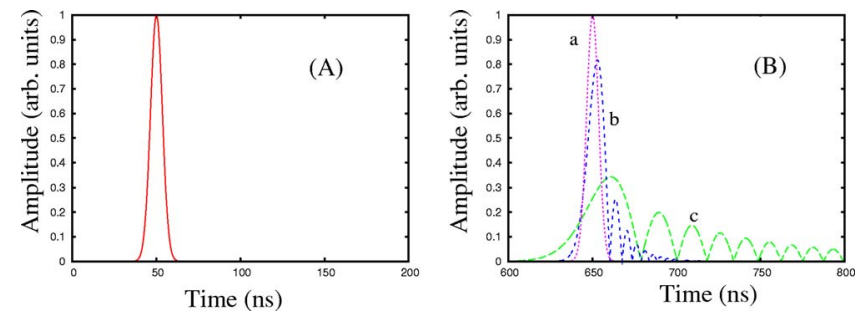

FIG. 3. (Color online) (A) Input pulse ( $T=5 \mathrm{~ns}$ ) is shown. (B) Delay time $\tau_{\text {delay }}=k \eta \gamma L / 2 \Omega^{2}=0.65 \mu \mathrm{s}$. The output pulses corresponding to different driving power are shown: (a) $\Omega T=5$, (b) $\Omega T=25$, (c) $\Omega T=100$.

magnetic field induced inhomogeneous broadening.

It is clear from Eq. (3) that the group velocity is positive if $\Omega>1 / T$, where $T$ is the duration of the pulse. Thus, in order to have a delay of the probe pulse, a necessary condition is $\Omega T>1$.

In order to see the distortion of pulses propagating through the EIT medium, we consider the propagation of a Gaussian probe pulse $\Omega_{p}(t, z)$, whose spectrum is also Gaussian, i.e.,

$\Omega_{p}(t, z=0)=\Omega_{p 0} \exp \left[-\frac{t^{2}}{2 T^{2}}\right], \quad \Omega_{p}^{\omega}=\frac{T \Omega_{p 0}}{\sqrt{2 \pi}} \exp \left[-\frac{T^{2} \omega^{2}}{2}\right]$.

The wave number at frequency $\nu_{p}$ is given by

$$
k\left(\nu_{p}\right)=\frac{\nu_{p}}{c} \sqrt{1+\chi\left(\nu_{p}\right)} \simeq \frac{\nu_{p}}{c}\left[1+\frac{\chi\left(\nu_{p}\right)}{2}\right],
$$

and the probe pulse at the distance $z$ can be calculated by

$$
\Omega_{p}(t, z)=\frac{T \Omega_{p 0}}{\sqrt{2 \pi}} \int_{-\infty}^{+\infty} d \omega \exp \left[-i \omega t-\frac{T^{2} \omega^{2}}{2}+i k(\omega) z\right] .
$$

For large enough Rabi frequencies the atomic susceptibility is given by

$$
\chi^{\prime}\left(\nu_{p}\right) \simeq \frac{-\eta \gamma \Delta_{p}}{|\Omega|^{2}} .
$$

Therefore, at a position $z$, the delayed probe pulse is given by

$$
\Omega_{p}(t, z)=\Omega_{p 0} \exp \left[-\frac{\left(t-z / v_{g}\right)^{2}}{2 T^{2}}\right],
$$

where the group velocity of the optical pulse is $v_{g} \simeq 2|\Omega|^{2} / k \eta \gamma$ (see Ref. [7]), and the delay time is given by $\tau_{\text {delay }}=L / v_{g}=k \eta \gamma L / 2|\Omega|^{2}$. Taking into account that $\Omega T>1$, a good estimate for the delay time is

$$
\tau_{\text {delay }}<k \eta L \gamma T^{2} / 2 \text {. }
$$

Thus a high intensity driving field is needed to obtain significant delay for short pulses (see Fig. 3). For substantial delay times, the atomic density should also be large. For $N=10^{14} \mathrm{~cm}^{-3}, L=1 \mathrm{~cm}, \lambda \simeq 1 \mu \mathrm{m}$, we obtain 


$$
\tau_{\text {delay }}<10^{12} T^{2} \text {. }
$$

Thus, for $T=10^{-9} \mathrm{~s}$, the delay time is of the order of a few $\mu \mathrm{s}$, but for $T=1 \mathrm{ps}$, the delay time is just $1 \mathrm{ps}$. We also note that the required power for the picosecond time scale is six orders of magnitude higher than that for nanosecond pulses.

To extend this technique to shorter time scales we suggest the setup shown in Fig. 1. Due to the spectral dispersion of the prisms, the probe beam has an angular spread after the first prism. The second prism has the same frequency dispersion, but its dispersion has the opposite sign relative to the first one. Thus after the second prism the optical beam components become parallel and there is a one-to-one correspondence between the $x$ position and the frequency of the probe and drive fields. As discussed earlier, the inhomogeneous magnetic field shifts the atomic levels such that the probe and the drive fields are in both one-photon and two-photon resonances for atoms at all locations inside the medium. In the limit of infinite spectral resolution, prisms separate different frequencies of the input pulse, and then these frequencies propagate through the EIT medium. For each frequency, the EIT medium produces a time delay given by $L / v_{g}$. This results in the delay for the whole pulse which is restored after the second pair of prisms (see Fig. 1). The important improvement due to this scheme is that we relax the condition on the strength of the driving field whose role is limited to providing only EIT for a particular frequency. There is no requirement on the driving field to provide a linear dispersion for the range of frequencies corresponding to the pulse spectrum.

However, in practice there is not infinite resolution of frequencies at the input plane $z=0$. Instead, due to the prism dispersion, there is a frequency distribution at each point on the input plane whose width is of course considerably lower than the spectral width of the original pulse. We next calculate the spectral width of the field at a given point in the input plane (after passage through the double lens system of Fig. 1).

We assume, for simplicity, that the input pulse has a Gaussian spatial distribution of width $d$ perpendicular to the direction of propagation, as well as a Gaussian time profile corresponding to its duration $T$, i.e.,

$$
\Omega_{p}(t, x, z=0)=\Omega_{p 0} \exp \left[-\frac{t^{2}}{2 T^{2}}-\frac{x^{2}}{2 d^{2}}\right] .
$$

As shown in Fig. 2(A), the field at an arbitrary location $x_{0}$, corresponding to frequency $\omega_{0}$ in the plane $z=0$, is obtained from a spatial distribution of the original pulse before the first pair of prisms. The field of frequency $\omega_{x}$ coming from the $x$ position of the input pulse at $x_{0}$ is related to $\omega_{0}$ via $x=\alpha L_{p}\left[d n\left(\omega_{0}\right) / d \omega_{0}\right]\left(\omega_{x}-\omega_{0}\right)$, where $L_{p}$ is the distance between the first two prisms [8] (see Fig. 2). The time dependence of the pulse at the location $x_{0}$ is given by adding all frequencies over the transverse distribution of the beam. In Fig. 2, we show five positions in the D-region, the laser pulse at each position has a central frequency and some spread of frequencies that comes from the distribution of the optical beam in the $x$-direction. Then the probe pulse at a location $x_{0}$ with central frequency $\omega_{0}$ is given by

$$
\begin{aligned}
\Omega_{\omega_{0}} & =\frac{T \Omega_{p 0}}{\sqrt{2 \pi}} \int d \omega_{x} \exp \left[-\left(\tau^{2}\left(\omega_{x}-\omega_{0}\right)^{2}+T^{2} \omega_{x}^{2}\right) / 2-i \omega_{x} t\right] \\
& =\frac{T \Omega_{p 0} \exp \left[-\left(T^{2} \tau^{2} \omega_{0}^{2}-t^{2}-2 i \omega_{0} \tau^{2} t\right) / 2\left(T^{2}+\tau^{2}\right)\right]}{\sqrt{T^{2}+\tau^{2}}}
\end{aligned}
$$

where we have introduced $\tau^{2}=\alpha^{2} L_{p}^{2}\left(d n\left(\omega_{0}\right) / d \omega_{0}\right)^{2} / d^{2}$. It is clear that, in the limit $d \rightarrow 0$, we have $\tau \rightarrow \infty$ and this corresponds to infinite spectral resolution.

Thus, in the dispersion region, the pulse at a given frequency $\omega_{0}$ has a time duration $\sqrt{T^{2}+\tau^{2}}$ where $\tau$ is related to the dispersion of the prism's system $\beta^{\prime}$. After passing through the EIT medium each pulse at a given frequency experiences a delay $\tau_{d}=L / v_{g}$, if the Rabi frequency of the drive field satisfies $\Omega \sqrt{T^{2}+\tau^{2}} \simeq \Omega \tau \gg 1$ instead of $\Omega T \gg 1$. Here we see that the prism arrangement allows us to substantially relax the condition on the driving field.

On combining all the frequency components after passage through the medium, we therefore reproduce a short pulse with duration equal to initial time duration $T$ but shifted by $\tau_{d}$, i.e.,

$$
\Omega_{p}(t, z)=\int d \omega_{0} \Omega_{p}\left(t-\tau_{d}, x_{0}, z\right)=\Omega_{p 0} \exp \left[-\frac{\left(t-\tau_{d}\right)^{2}}{2 T^{2}}\right] .
$$

We note that different frequency components will acquire linear and nonlinear phase shifts while passing through the EIT medium and appropriate phase shifetres will be required to compensate these phase shifts.

The duration of the pulse is determined by the spectral width, which is determined by the inhomogenous broadening that we introduce via the inhomogeneous magnetic field. We note that, locally, the medium is always resonant with the probe and drive fields. The delay time is determined by the density of atoms

$$
\tau_{\text {delay }}=\frac{L}{v_{g}}=\frac{k \eta \gamma L}{2|\Omega|^{2}} .
$$

There should be no absorption if $\Omega \gg 1 / \tau$. For the parameters $N=10^{14} \mathrm{~cm}^{-3}, \Omega \simeq 10 \gamma, 1 / \tau \simeq \gamma=2 \times 10^{7} \mathrm{~s}^{-1}, L=1 \mathrm{~cm}, \lambda$ $\simeq 1 \mu \mathrm{m}$, we have

$$
\tau_{\text {delay }}=\frac{k \eta \gamma L}{2|\Omega|^{2}} \simeq 10 \mu \mathrm{s}
$$

i.e., even for a $T=10$ ps pulse the delay time is of the order of $10 \mu \mathrm{s}$. In addition, for short pulses having a broad spectrum there is a requirement on the magnetic field. That is, one needs to create the magnetic field with a gradient matching the dispersion in the prism system so that at each position in the D-region the probe field is at resonance. The total shift of the atomic level should correspond to the spectrum of the short pulse $\Gamma=1 / T$. This introduces the condition on the atomic configuration, namely the level $b$ should be able to move linearly with the magnetic field. For example, this condition can be met in $\mathrm{Ca}$ atom where the states $P_{0}, P_{1}$, separated by $52 \mathrm{~cm}^{-1}$, and the excited 
state $S_{1}$ form the three-level $\Lambda$ configuration suitable for our purposes. Thus, for a $10 \mathrm{ps}$ pulse, the change of the magnetic field needs to be about $1 \mathrm{~T}$ per atomic cell. This gradient can be created by using just a simple wire with electric current $I=10^{2} \mathrm{kA}$ (the cell has length $l=10 \mathrm{~cm}$ and one side of the cell is at the distance of $r_{1}=1 \mathrm{~cm}$ from the wire and the second side at $r_{2}=11 \mathrm{~cm}$ ), or using antiHelmholtz coils.

In conclusion, we have suggested and theoretically analyzed a system that can potentially provide delays for broadband optical pulses. Applications of these results hold substantial promise ranging from radar systems and femtosecond pulse shaping techniques to quantum storage for photons having very short coherence times.

We thank Philip Hemmer for helpful discussions. We also gratefully acknowledge the support from the Office of Naval Research, the Air Force Office of Scientific Research, the Defense Advanced Research Projects Agency-QuIST, Texas A\&M University Telecommunication and Information Task Force (TITF) Initiative, the Robert A. Welch Foundation, Horace C. Hearne Foundation, the Advanced Research Projects Agency, the National Security Agency, and the Army Research Office.
[1] T. Kamiya, F. Saito, O. Wada, and H. Yajima, Femtosecond Technology (Springer, Berlin, 1999).

[2] S. E. Harris and L. V. Hau, Phys. Rev. Lett. 82, 4611 (1999); P. C. Ku, C. J. Chang-Hasnain, and S. L. Chuang, Electron. Lett. 38, 1581 (2002).

[3] S. E. Harris, J. E. Field, and A. Imamoglu, Phys. Rev. Lett. 64, 1107 (1990).

[4] M. O. Scully and M. S. Zubairy, Quantum Optics (Cambridge University Press, Cambridge 1997).

[5] L. V. Hau, S. E. Harris, Z. Dutton, and C. H. Behroozi, Nature (London) 397, 594 (1999); M. M. Kash, V. A. Sautenkov, A. S. Zibrov, L. Hollberg, G. R. Welch, M. D. Lukin, Y. Rostovtsev, E. S. Fry, and M. O. Scully, Phys. Rev. Lett. 82, 5229 (1999); D. Budker, D. F. Kimball, S. M. Rochester, and V. V. Yashchuk, ibid. 83, 1767 (1999).

[6] A. V. Turukhin, V. S. Sudarshanam, M. S. Shahriar, J. A. Musser, B. S. Ham, and P. R. Hemmer, Phys. Rev. Lett. 88,
023602 (2001); M. S. Bigelow, N. N. Lepeshkin, and R. W. Boyd, ibid. 90, 113903 (2003).

[7] A. B. Matsko, O. Kocharovskaya, Y. Rostovtsev, G. R. Welch, A. S. Zibrov, M. O. Scully, Adv. At., Mol., Opt. Phys. 46, 191 (2001).

[8] This relation is obtained for thin prisms. The general case can be found in M. Born and E. Wolf, Principles of Optics (Cambridge University Press, New York, 1999).

[9] Z. Dutton, M. Bashkansky, M. Steiner, and J. Reintjes, Proc. SPIE 5735, 115 (2005).

[10] Z. Deng, D.-K. Qing, P. Hemmer, C. H. R. Ooi, M. S. Zubairy, and M. O. Scully (unpublished).

[11] A. B. Matsko, D. V. Strekalov, and L. Maleki, Opt. Express 13, 2210 (2005).

[12] R. W. Boyd, D. J. Gauthier, A. L. Gaeta, and A. E. Willner, Phys. Rev. A 71, 023801 (2005). 